\title{
Interpreting the drying kinetics of a soil using a macroscopic thermodynamic non-equilibrium of water between the liquid and vapour phase
}

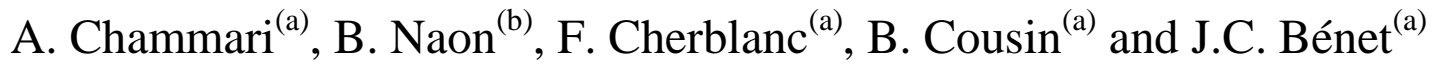 \\ (a) L.M.G.C., Université Montpellier 2, place Eugène Bataillon, 34095 Montpellier cedex 05, France. \\ (b) IUT - Université Polytechnique de Bobo-Dioulasso, 01 BP 1091 Bobo-Dioulasso, Burkina Faso.
}

\section{Shortened title}

Thermodynamic non-equilibrium during the drying of a soil.

\begin{abstract}
Two preliminary experiments show that a non-equilibrium situation can be easily encountered during a natural drying process. This leads us to reconsider the thermodynamic local equilibrium assumption, and propose a macroscopic two-equation model that takes into account mass exchange kinetics between the liquid and vapour phase. Numerical simulation of this theoretical model is then compared to experimental drying kinetics of soil columns. The discrepancies observed between the theoretical prediction and the experimental results are discussed. This contribution emphasizes the importance of such non-equilibrium phenomenon when modelling water transport in hygroscopic porous media.
\end{abstract}

\section{Keywords}

Drying kinetics - porous media - phase change - non-equilibrium - natural attenuation

\section{Introduction}

Drying kinetics generally displays two phases. It is globally accepted that liquid evaporates at the surface during the first phase, referred to as the constant rate phase. The second phase is called the diffusion phase and drying kinetics is controlled by diffusion mechanisms within the material [1]. When modelling this second phase, the state variable chosen is generally the liquid water content. Since the medium includes a 
gas phase consisting of moist air, using a single variable for describing the moisture state implicitly assumes the thermodynamic equilibrium between the liquid water and its vapour. This hypothesis implies sufficiently rapid mass exchanges between phases to maintain at all times the thermodynamic equilibrium characterised by the equality of chemical potentials between the liquid and its vapour [2-6]. However, this hypothesis seems to be called into question by experiments about liquid-gas phase change in porous media that suggest that the establishment of equilibrium is not instantaneous. This applies to soil containing water or heptane at various temperatures $[7,8]$. The liquid-vapour equilibrium assumption makes tricky the interpretation of drying kinetics of certain soils such as silt, since the water state can evolve from the funicular state, characterised by a continuous flowing liquid phase, to the hygroscopic state, where the water is adsorbed on the solid phase. As the water content decreases, the liquid phase is no longer continuous and movement in the liquid phase cannot be envisaged. As shown below, the water state of a moist sample subjected to a water content gradient or gravity in a saturated atmosphere does not change. Furthermore, as the medium is not yet hygroscopic, the vapour partial pressure profiles are uniform and movement by diffusion of vapour cannot take place.

Therefore, we propose to discard the hypothesis of thermodynamic equilibrium between liquid water and its vapour when providing the interpretation of drying kinetics of a soil in the pendular and hygroscopic states. Two state variables, the liquid water content and the partial pressure of vapour in the pores, are used to represent the system $[8,9]$. Vapour diffusion and liquid-vapour phase change are considered as the main phenomena. To emphasize our point of view, two preliminary experiments are first described. It aims to qualitatively demonstrate the existence of liquid-vapour nonequilibrium inside a soil sample submitted to drying conditions. This is followed by an experimental water phase change characterization. Then, a set of drying experiments of soil columns is presented, allowing a macroscopic comparison with the theoretical prediction.

\section{Preliminary experiments}

Theoretical aspects developed in this contribution are based on the nonequilibrium assumption. To justify this point of view, two preliminary experiments are 
presented below; the first one brings some intermediates conclusions, while the second one demonstrates the existence of a non-equilibrium situation during a natural drying process.

\subsection{Materials and methods}

The material under investigation is a natural soil (clayey silty sand), coming from the riversides of the Hérault river (south of France). Its real mass density is $\rho_{\mathrm{s}}=2650 \mathrm{~kg} \cdot \mathrm{m}^{-3}$. The mineralogical analysis based on X-ray diffraction techniques has revealed a small clayey fraction $(\sim 10 \%)$ and a negligible organic content. The lower limit of the funicular domain is around $w=13 \%$ [10]. Below this water content, the liquid phase is no longer continuous and the relative permeability is zero [10]. Regarding to the hygroscopic domain, the desorption isotherm is shown in Figure 1. It can be seen that hygroscopic effects are observed with a water content lower than $w=5 \%$. For indication the saturation is obtained for a water content of $w=29 \%$.

In these preliminary experiments, soil samples were prepared with a uniform $10 \%$ water content. The soil was first dried at $105^{\circ} \mathrm{C}$ during 24 hours. The required amounts of soil and demineralized water were added using a high-precision scale $\left(10^{-4} \mathrm{~g}\right)$. After mixing, it was stored in a waterproof container for 24 hours to ensure the homogeneity of the water content. Then, the wet soil was compacted in a cylindrical ring by means of a hydraulic press in order to reach a dry density of $1500 \mathrm{~kg} . \mathrm{m}^{-3}$, which corresponds to a porosity of $43 \%$. In each cases presented below, the liquid water content, $w$, was measured by differential weighing after 24 hours drying at $105^{\circ} \mathrm{C}$.

\subsection{First Experiment}

The soil samples were disposed in a controlled drying atmosphere at $T=30^{\circ} \mathrm{C}$. The relative humidity was regulated at $R H=33 \%$ using a saturated magnesium chloride solution. The sample geometry was chosen so that to consider a one-dimensional mass transfer along the vertical $z$-axis (Fig. 2). Three thermocouples introduced in the soil sample during compaction did not reveal any temperature gradient or variation during the drying phases. The drying kinetics was recorded by differential weighing (Fig. 3). A destructive method was applied to some samples to determine the water content profiles at different times (Fig.4). Kinetics and profiles evolutions show some classical appearances. 
This experiment was repeated in the same conditions, except that at time $t_{0}$, when the average water content is around $5.5 \%$, the magnesium chloride solution was replaced by pure water in order to impose a relative humidity $R H=100 \%$. Consequently, the partial pressure of vapour $P_{v}$ in the soil is set to its saturated value $P_{v s}$. Since the soil was not yet in a hygroscopic state, this partial pressure corresponds to the equilibrium vapour pressure $P_{\text {veq }}$. The instantaneous stabilization of the average water content (Fig. 3) and of the water content profiles (Fig. 5) was then observed in spite of the strong water content gradient. It was noticed that if the sample was put back to the initial experimental conditions $(R H=33 \%)$, the drying process starts again and the kinetics and profiles measured exhibit their usual pattern.

The following features were deduced from this experiment:

i- $\quad$ The application of the equilibrium relative humidity at the outer surface of the sample stops any mass transfer.

ii- When the surrounding atmosphere is stable and saturated, the water content gradient and the gravity forces are unable to cause any liquid phase flow. This confirms that the water was in a pendular state.

iii- A change in the $R H$ boundary condition was instantaneously transmitted to the whole sample, since the characteristic time of vapour diffusion process is negligible if compared to the characteristic time of this drying experiment.

\subsection{Second Experiment}

In this second stage, the same material is considered. The samples were compacted in three parts (A, B and C) and assembled leaving a $1 \mathrm{~mm}$ air space between them (Fig. 6) [11]. They were placed in a drying atmosphere regulated at $T=30^{\circ} \mathrm{C}$ and $R H=19.5 \%$. The destructive measure consists in separating the three parts and weighing each of them. Thermocouples placed in each part did not reveal any significant temperature difference during one experiment. It can be seen in Figure 7 that the drying of the central part $A$ starts at the beginning of the experiment whereas the soil in parts $B$ and $C$ were not yet in a hygroscopic state $(t<45 \mathrm{~min})$.

This experiment shows that the liquid phase flow is not the only mechanism involved during drying. Indeed, if this was the case, part A could not dry since the air space constitutes a liquid phase discontinuity that prevents any liquid phase flow. 
Therefore, the liquid water present inside the soil can only be extracted through vapour diffusion, which necessitates a liquid-gas phase change.

Based on the conclusions pointed up in the last section, it can be asserted that drying of the central part $A$ can occurs only if non-equilibrium exists between the vapour in the air spaces and the water at the surface of $A$. This implies that there is not liquid-vapour equilibrium throughout the parts $B$ and $C$. Thereby, even with the slow natural drying process observed in parts $B$ and $C$, non-equilibrium situations are easily encountered.

These preliminary experiments lead us to reconsider the local equilibrium assumption between the liquid water and its vapour. This hypothesis is discarded; therefore, a non-equilibrium liquid-gas vaporization law is introduced to describe the kinetics of the overall drying of a soil column. Next section focuses on the development of a water and vapour transport model including the phase change phenomenon.

\section{Water transport in a non-saturated soil}

A natural soil can be idealized by a triphasic porous medium by considering a solid phase, a liquid phase and a gaseous phase. Regarding to the drying process, the gaseous phase consists of two components: dry air and water vapour. As discussed in the last section, the model proposed in this work relies on the two following assumptions:

- The liquid phase is adsorbed on the solid phase and immobile, meaning that liquid surface diffusion is not taken into account. Even if surface diffusion can be observed at the microscopic scale, the kinetics of such phenomenon becomes negligible when dealing with macroscopic transport problem.

- The total gas pressure is constant and uniform, since the convective transport in the gas phase is negligible. Actually, this means that the gas permeability is large enough to assume that any pressure gradient will be instantaneously equilibrated when compared to the other transport phenomena.

Therefore, two elementary phenomena are considered: liquid-gas phase change of water and vapour diffusion in the gas phase. Associated to these phenomena, the state variables are the water content $w[\%]$ defined as the ratio between the apparent mass 
densities of liquid and solid, and the vapour partial pressure in the gas phase $P_{v}[\mathrm{~Pa}]$. Then, the mass balance for the liquid phase is written:

$$
\frac{\partial}{\partial t} w=-\frac{J}{\rho_{s}}
$$

while the mass balance for the vapour constituent in the gas phase is given by:

$\eta_{g} \frac{\partial}{\partial t} P_{v}=\frac{\partial}{\partial x}\left(D_{v s} \frac{\partial}{\partial x} P_{v}\right)+\frac{R}{M} J$

where $\rho_{s}$ is the apparent mass density of the solid phase, $\eta_{g}$ is the volume fraction of the gas phase, $D_{v s}$ is the effective diffusion coefficient of vapour in the soil, $R$ is the perfect gas constant and $M$ is the molar mass of water. The mass exchange term $J\left[\mathrm{~kg} . \mathrm{s}^{-1} \cdot \mathrm{m}^{-3}\right.$ ] represents the rate of water phase change from the liquid to the gas phase.

From thermodynamic considerations, it can be shown that the volumetric rate of phase change $J\left[\mathrm{~kg} \cdot \mathrm{m}^{-3} \cdot \mathrm{s}^{-1}\right]$ is proportional to the water chemical potential difference between the liquid and vapour states [12-17]. A detailed development of this phase change theoretical relation has been given by Bénet et al. [12]. Thus, only the main results are recalled here. The phase change rate is expressed as a function of the vapour partial pressure by:

$$
J=J_{e q}-L \frac{R}{M} \ln \frac{P_{v}}{P_{v e q}}
$$

It is written as the sum of an equilibrium part, $J_{e q}$, and a non-equilibrium part. The equilibrium part accounts for the phase change resulting from temperature variations while the liquid water remains in equilibrium with its vapour. For instance, it represents the water quantity that evaporates during a temperature raise to maintain the saturating vapour pressure in the gas phase. It generally relates to slow or quasi-static phenomena. On the contrary, the non-equilibrium part characterizes the response of the system to a non-equilibrium situation $[12,13,15]$. This non-equilibrium results from a chemical potential difference between the liquid water and its vapour. However, it is better represented by a deflection of the vapour pressure $P_{v}$ with respect to its equilibrium value $P_{\text {veq }}$. The vapour pressure at equilibrium $P_{\text {veq }}$, is defined as the product of the saturating vapour pressure $P_{v s}$ multiplied by the water activity $a$. Usually, the water activity is directly determined from the experimental sorption isotherm. In the theoretical law proposed (Eq. 3), the ratio of the vapour partial pressure to its 
equilibrium value can be interpreted as the thermodynamic force that governs the phase change.

The phenomenological coefficient $L\left[\mathrm{~kg} . \mathrm{K} . \mathrm{s} . \mathrm{m}^{-1}\right]$, introduced in this relation, should depends on the state variables, such as the water content $w$ and the temperature $T$, and on the nature of the soil. This coefficient must be determined experimentally, and has been the focus of several works $[12,13,15,17]$. An original experimental device has been developed for this purpose. It allows analysing the return back to equilibrium of a soil sample subjected to non-equilibrium conditions. This non-equilibrium situation is caused by, first, extracting the gas phase of the soil sample, and then, replacing it by dry air, what results in a macroscopic thermodynamic non-equilibrium between the liquid phase and its vapour. Thus, the dependence of the phase change coefficient $L$ on several physical variables, such as the temperature $T$, the water content $w$, the total gas pressure $P_{g}$, has been experimentally investigated $[15,17]$. The influence of the nature of the liquid phase and of the texture of the soil has also been underlined.

From a large set of experimental data carried out in isothermal conditions with pure water in clayey silty sand, Lozano et al. [17] have provided a complete model of the phase change coefficient. Its variations as functions of the water content and the vapour partial pressure are characterised by 3 coefficients $\left(L_{e q}, k, r\right)$ through the following expressions:

- close to equilibrium: $\quad r \leq \frac{P_{v}}{P_{v e q}} \leq 1 \quad L=L_{e q}$

- far from equilibrium: $\quad 0<\frac{P_{v}}{P_{v e q}}<r \quad L=L_{e q}+k\left(r-\frac{P_{v}}{P_{v e q}}\right)$

The neighbourhood of an equilibrium situation, i.e., when the vapour partial pressure $P_{v}$ is close to its equilibrium value, corresponds to the validity domain of the linear thermodynamics of irreversible processes, and a constant phenomenological coefficient is observed. Outside of this domain, i.e., far from equilibrium, an affine dependence on the vapour partial pressure is obtained, and the phase change rate is highly increased.

The influence of the water content $w$ on the three parameters $\left(L_{e q}, k, r\right)$ is presented in Figures 8 to 10. Some bell-shaped curves are generally observed, where the maximum around $7 \%$ is roughly the upper limit of the hygroscopic domain. Above this 
maximum, the phase change rate decreases since the liquid-gas interface reduces. For water content greater than $12 \%$, the gas phase is occluded and phase change cannot be activated. Below the maximum, when hygroscopic effects become predominant, the intensity of solid-liquid interactions increases in the adsorbed layers. The supplementary energy required for water desorption decreases the phase change rate, leading to lower values of the coefficient.

Therefore, a complete two-equation model of water and vapour transport through a hygroscopic soil has been proposed. Using the physical characteristics identified on a centimetric-scale soil sample, numerical simulation will be compared to macroscopic experimental drying kinetics in the next section.

\section{Self drying of a soil at low water content}

In order to discuss the validity of our non-equilibrium assumption, the theoretical model presented in the last section will be used to analyse a natural drying kinetics. First, the drying experiments are described, then, the numerical implementation is briefly presented, and finally the comparison and useful discussions are provided.

\subsection{Experiments}

The same material characterized in the last section is used to make the soil columns, i.e., a clayey silty sand with a real mass density of $\rho_{s}{ }^{*}=2650 \mathrm{~kg} . \mathrm{m}^{-3}$. The wet soil was compacted in a PVC tube to reach a solid apparent mass density of $\rho_{s}=1500 \mathrm{~kg} \cdot \mathrm{m}^{-3}$, which corresponds to a porosity of $43 \%$. Sample dimensions were $10 \mathrm{~cm}$-height and $8.14 \mathrm{~cm}$-diameter. The upper surface is in contact with air, while the lower surface is hermetically closed. The initial water content of the soil is fixed at $w=8 \%$. Then, the soil samples are placed in a regulated drying atmosphere at controlled temperature $T=30^{\circ} \mathrm{C}$, and relative humidity $R H=30 \%$. Columns were weighed at regular time steps to determine the average water content leading to the drying kinetics plotted in Figure 12. The nine experimental kinetics were achieved with a good reproducibility [8]. For large times, an asymptotic water content is observed, $w=2 \%$, which corresponds to the equilibrium value given by the desorption isotherm.

\subsection{Theoretical Model and Numerical Simulation}


Since the temperature is controlled at $T=30^{\circ} \mathrm{C}$, the equilibrium phase change, $J_{e q}$, accounting for temperature variations can be discarded. Thus, the phase change relation (Eq. 3) can be introduced in the two-equation model (Eqs 1-2) to obtain

$$
\begin{aligned}
& \frac{\partial}{\partial t} w=L \frac{R}{\rho_{s} M} \ln \frac{P_{v}}{P_{v e q}} \\
& \eta_{g} \frac{\partial}{\partial t} P_{v}=\frac{\partial}{\partial x}\left(D_{v s} \frac{\partial}{\partial x} P_{v}\right)-L \frac{R^{2}}{M^{2}} \ln \frac{P_{v}}{P_{v e q}}
\end{aligned}
$$

where the phenomenological coefficient $L$ non-linearly depends on the variables $w$ and $P_{v}$ through the experimental correlations presented in Figures 8 to 10. The equilibrium vapour pressure $P_{v e q}$ is calculated using the desorption isotherm curve given in Figure 1

$$
P_{v e q}=a(w) P_{v s}\left(T=30^{\circ} \mathrm{C}\right)
$$

The gas volume fraction $\eta_{g}$ will slightly increase as the water content $w$ decreases by the following expression

$$
\eta_{g}=1-\frac{\rho_{s}}{\rho_{s}}-w \frac{\rho_{s}}{\rho_{l}}
$$

To take into account the soil tortuosity, the effective diffusion coefficient is weighted according to the expression proposed by Penman [18-20]

$D_{\mathrm{vs}}=0.66 \eta_{g} D_{v a}$

where the diffusion coefficient of vapour in air at $T=30^{\circ} \mathrm{C}$ is $D_{v a}=2.62 \mathrm{~m} \cdot \mathrm{s}^{-2}$.

For numerical simulation, these equations are discretized using a one-dimensional regular mesh, where the unknowns ( $w$ and $P_{v}$ ) are located at the centre of grid blocks. Temporal integration is performed based on an implicit scheme to ensure numerical stability. This problem is highly non-linear, mainly due to the complex dependence of the coefficient $L$ on the unknowns $w$ and $P_{v}$. Thus, a Newton-Raphson method ensures an accurate convergence for a moderate time step.

According to the experimental setup, the boundary conditions were a gas-phase no-flow condition on the lower surface of the sample,

$$
\left.\frac{\partial}{\partial t} P_{v}\right|_{z=0}=0
$$

and a imposed vapour pressure on the upper surface corresponding to relative humidity, $R H=30 \%$, of the drying atmosphere

$$
\left.P_{v}\right|_{z=H}=R H \times P_{v s}\left(T=30^{\circ} \mathrm{C}\right)
$$


The initial water content is imposed at $w=8 \%$, what corresponds to an initial equilibrium vapour pressure given by

$$
\left.P_{v}\right|_{t=0}=P_{v s}\left(T=30^{\circ} \mathrm{C}\right) \quad \text { since the activity is } \quad a(w=8)=1
$$

Eventually, the simulated kinetics is plotted on Figure 12 for comparison. Associated discussions are provided in the next section.

\subsection{Results}

While comparing the theoretical prediction with the experimental drying kinetics (Fig. 12), the agreement is not very acceptable. Indeed, estimated drying times are twice as large as those observed experimentally. However, this a priori conclusion should be reconsidered and discussed in detail.

First, it must be recalled that the comparison given here is done without any adjustable parameters or curve-fitting technique. Every physical characteristic has been determined using an independent experimental procedure. In particular, the effective diffusivity in soil is estimated through an empirical model (Eq. 10) [18] that has not been validated in our case. Actually, the numerical drying kinetics is very sensitive to the value of this effective diffusivity. A higher diffusivity leads to some lower vapour pressure in the sample, which drastically enhances the water phase change based on equation (5). This parameter could have been adjusted from experimental kinetics to better suit the physical diffusion phenomenon. However, we prefer not to perform that estimation in order to fairly discuss our approach.

Secondly, the boundary condition numerically imposed (Eq. 12) is practically difficult to maintain accurately. A local relative humidity transducer disposed on the surface should give some response. Moreover, with respect to the phase change phenomenon, the upper boundary layer $(\sim 5 \mathrm{~mm})$ does not behave exactly the same as that present inside the sample. Indeed, destructive analyses of soil samples have shown a faster drying process of this upper layer. Further investigations are needed to improve this boundary condition and give some more valuable drying kinetics comparisons.

The cause of a macroscopic thermodynamic non-equilibrium has not yet been clearly established. However, the explanation should be sought at a smaller scale. A natural soil is an extremely heterogeneous medium at multiple scales. The clayey fraction creates some very fine porous aggregates $(\sim 0.1 \mu \mathrm{m})$ embedded in a larger 
heterogeneous structure made of sand grains $(\sim 100 \mu \mathrm{m})$ [21]. With clayey silt at low water content, a double-porosity structure is often encountered [22]. Moreover, even with a small clayey fraction, $10 \%$ in our case, about $90 \%$ of the water is stored in the clayey structure. At equilibrium, the liquid water is adsorbed in this complex system by some mechanisms that depend on the nature of the solid phase materials and their surface (clay, quartz, calcite ...) [21]. Thus, even if the second principle of thermodynamics imposes the uniformity of the water chemical potential in all its forms, its distribution should be very complex.

Any disturbance of this equilibrium by mass exchange with the environment will distort the uniformity of water chemical potential. This generally comes with temperature non-uniformities resulting from interface cooling. At the pore-scale, the reestablishment of equilibrium consists in the movement of liquid water, or vapour, from places with a high chemical potential to places with a low chemical potential. Local thermal transfers should also occur. These mechanisms are not instantaneous and the combination of them should account for the retardation times observed at the macroscopic scale.

Nevertheless, this experimental contribution shows that a non-equilibrium situation can be easily reached, even with the slow process of self drying. In this case, the nature of the solid phase plays a predominant role since it strongly depends on the hygroscopic characteristics of the porous medium.

\section{Conclusion}

When dealing with water transport in soils, discarding the assumption of a macroscopic thermodynamic equilibrium between the liquid and its vapour leads to the consideration of a vapour partial pressure deviation with respect to the equilibrium. This requires two independent variables to describe the water state in a soil, the liquid water content and the partial pressure of vapour. Therefore, the two associated mass balance equations are linked through a mass exchange term that represents the phase change phenomenon. A non-linear behaviour is experimentally observed as the phase change kinetics highly depends on the vapour partial pressure and on the liquid water content. 
This point of view replies to the challenges raised by the preliminary experiments described at the beginning of this paper. In some situations, and particularly with hygroscopic porous media, the local thermodynamic equilibrium assumption cannot be achieved. This could considerably modify the drying kinetics by emphasizing some different limiting processes.

The representation of a set of micro-scale phenomena using a unique macroscopic law corresponds to a classical upscaling approach. This point of view introduces some macroscopic coefficient that account for all the microscopic deviations from equilibrium. Nevertheless, the phenomenological approach proposed here, relies on an experimental determination of macroscopic coefficients, since the whole complexity of natural porous media is taken into account.

\section{References}

[1] Schünder, E.U. Drying of Porous Material during the Constant and the Falling Rate Period: A Critical Review of Existing Hypotheses, Drying Technology 2004, 22, $1517-1532$.

[2] Whitaker, S. Simultaneous Heat, Mass and Momentum Transfer in Porous Media: A Theory of Drying, Advances in Heat Transfer 1977, 13, Academic Press, New York, 119-203.

[3] Moyne, C.; Perre, P. Processes related to drying. Part I. Theoretical model, Drying Technology 1991, 9, 1135-1152.

[4] Perre, P.; Moyne, C. Processes related to drying. Part II. Use of the same model to solve transfers both in saturated and unsaturated porous media, Drying Technology 1991, 9, 1153-1179.

[5] Couture, F.; Fabrie, P.; Puiggali, J.R. An alternative choice for the drying variables leading to a mathematically and physically well described problem, Drying Technology 1995, 13, 519-550. 
[6] Prat, M. Recent advances in pore-scale models for drying of porous media, Chemical Engineering Journal 2002, 86, 153-164.

[7] Armstrong, J.; Frind, E.; McClellan, R. Non-equilibrium mass transfer between the vapor, aqueous, and solid phases in unsaturated soils during vapor extraction, Water Resources Research 1994, 30, 355-368.

[8] Chammari, A. ; Naon, B. ; Cherblanc, F. ; Bénet, J.C. Transfert d'eau en sol aride avec changement de phase, C. R. Mécanique 2003, 331, 759-765.

[9] Bénet, J.C.; Fras, G.; Jouanna, P. Changement de phase de l'eau dans un milieu poreux ; Application à l'étude du séchage d'un grain poreux, Euromech 194, Nancy, France, July 2-5, 1985.

[10] Saix, C.; Devillers, P.; El Youssoufi, S. Eléments de couplage thermomécanique dans la consolidation des sols non saturés, Canadian Geotechnical Journal 2000, 37, 110.

[11] Fras, G. Macroscopisation des transferts en milieux dispersés multiphasiques Application à l'étude de l'interface entre un milieu poreux et une athmosphère séchante, $\mathrm{PhD}$ thesis, 1989, University Montpellier 2, France.

[12] Bénet, J.C.; Jouanna, P. Phenomenological relation of phase change of water in a porous medium: experimental verification and measurement of the phenomenological coefficient. International Journal of Heat and Mass Transfer 1982, 25, 1747-1754.

[13] Bénet, J.-C.; Jouanna, P. Non équilibre thermodynamique dans les milieux poreux non saturés avec changement de phase. International Journal of Heat and Mass Transfer 1983, 26, 1585-1595.

[14] Kuiken, G.D.C. Thermodynamics for Irreversible Processes, Wiley, Chichester, 1994. 
[15] Ruiz, T.; Bénet, J.C. Phase Change in a Heterogeneous Medium: Comparison between the Vaporisation of Water and Heptane in an Unsaturated Soil at two Temperatures, Transport in Porous Media 2001, 44, 337-353.

[16] Bedeaux, D.; Kjelstrup, S. Irreversible thermodynamics - a tool to describe phase transitions far from global equilibrium, Chemical Engineering Science 2004, 59, $109-118$.

[17] Lozano, A.L.; Cherblanc, F.; Cousin, B.; Bénet, J.C. Experimental study and modelling of the water phase change kinetics in soils, European Journal of Soil Science 2008 (to be published).

[18] Penman, H.L. Gas and vapour movement in soil. 1: The diffusion of vapour through porous solids, Journal of Agricultural Science 1940, 30, 437-462.

[19] De Vries, D.A.; Kruger, A.J. On the Value of the Diffusion Coefficient of Water Vapor in Air, Proceedings of the International CNRS Conference, Transport Phenomena with Phase Change in Porous Media or Colloids, CNRS eds., 1966, Paris, $18-20$.

[20] Riddick, J.A.; Bunger, W.B.; Sakano, T.K. Organic solvent, physical properties and methods of purification, John Wiley and Sons, New York, 1986.

[21] Mitchell, J.K. Fundamentals of soil behaviour, John Wiley and Sons, New York, 1993.

[22] Delage, P.; Audiguier, M.; Cui, Y.J.; Howat, M. Microstructure of a compacted silty clay, Canadian Getotechnical Journal 1996, 33, 150 - 158. 


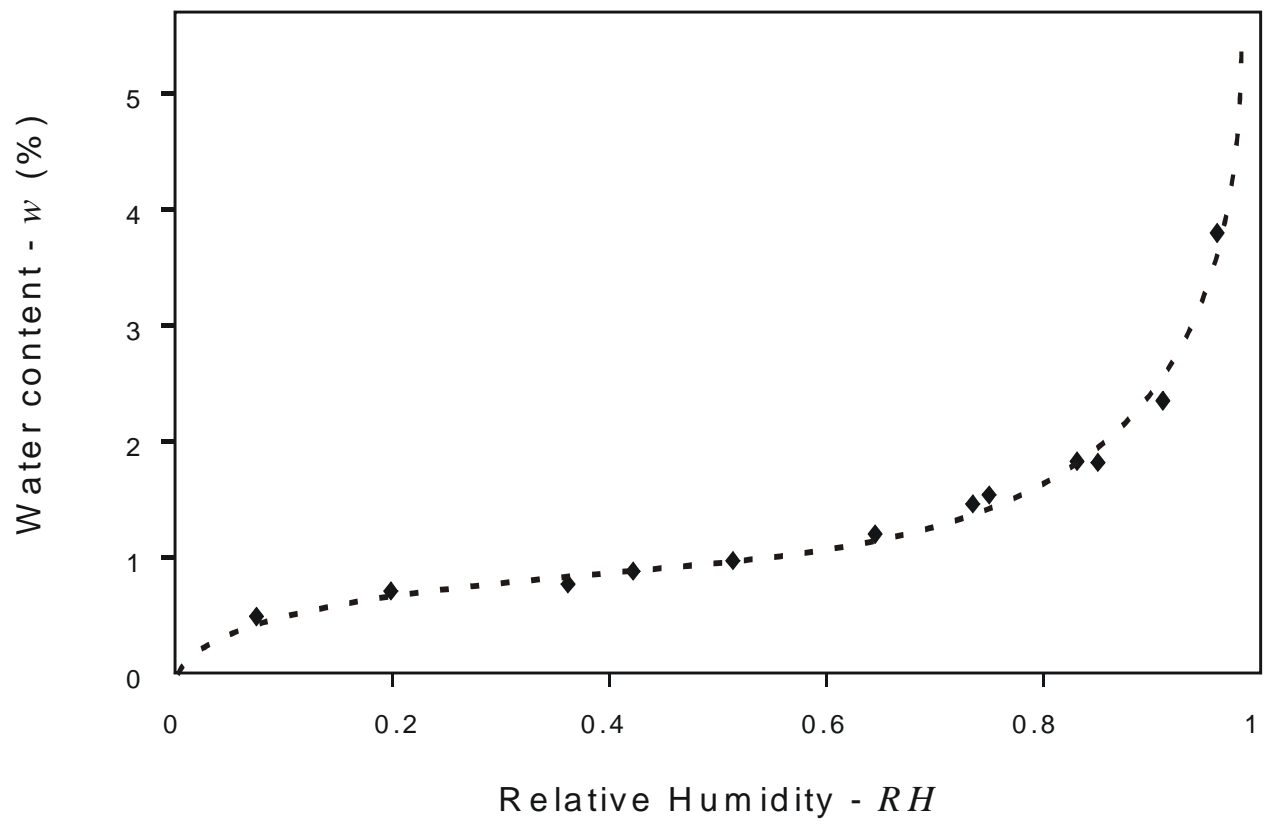

Figure 1: Desorption isotherm of the clayey silty sand at $T=30^{\circ} \mathrm{C}$, experimental points and fitted model. 


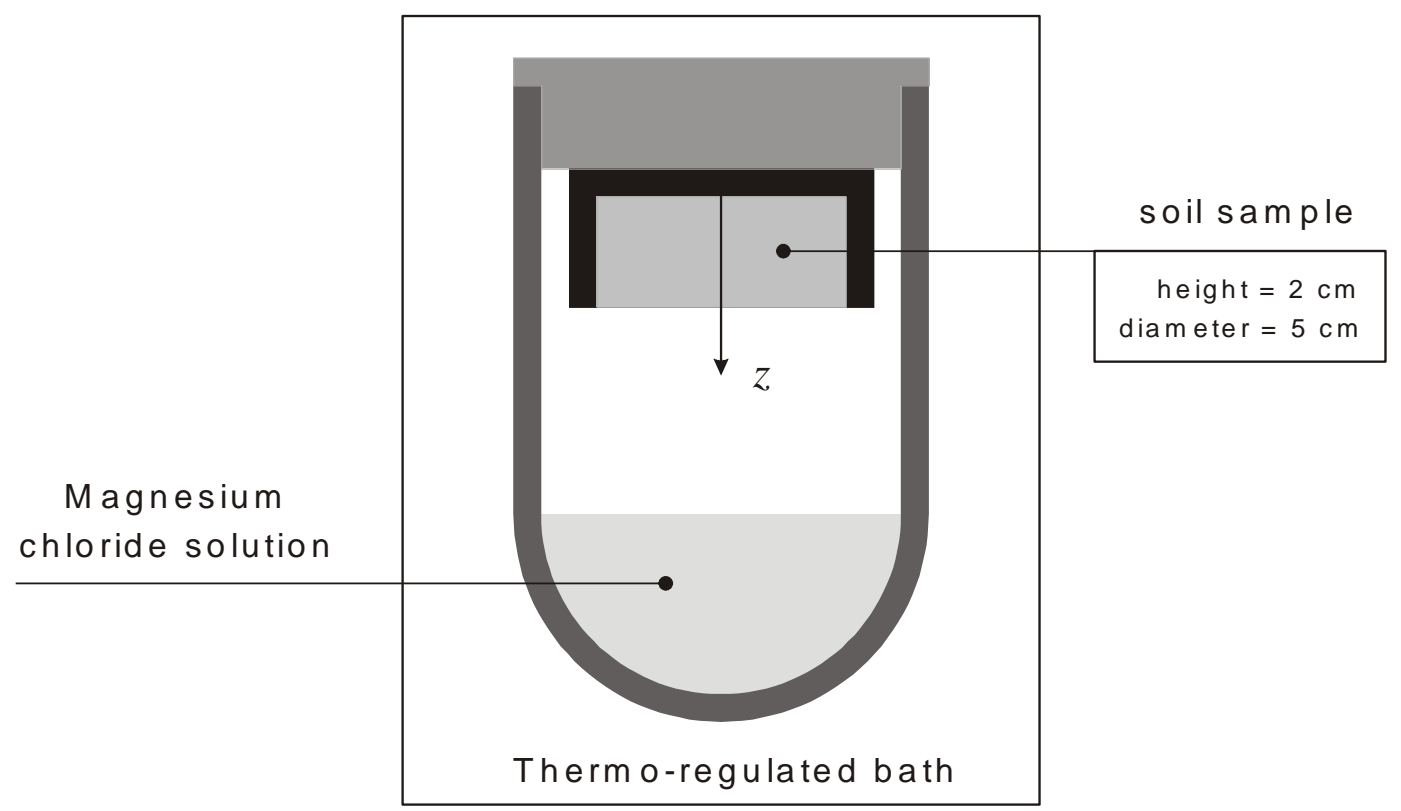

Figure 2: First preliminary experiment: Schematic view of the experimental device. 


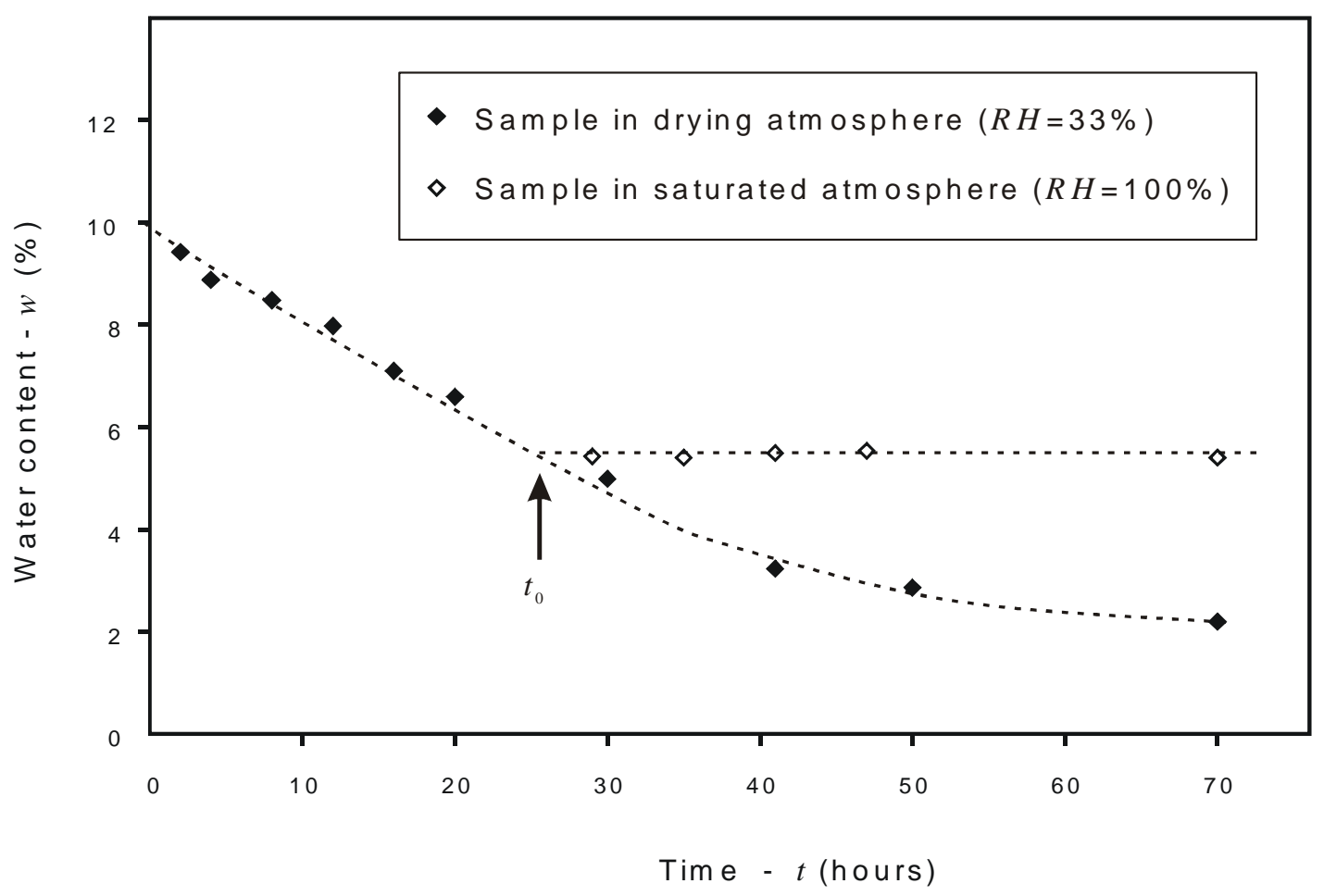

Figure 3: First preliminary experiment: Drying kinetics at $T=30^{\circ} \mathrm{C}$ and $R H=33 \%$ (black square) $-R H=100 \%$ (white square). 


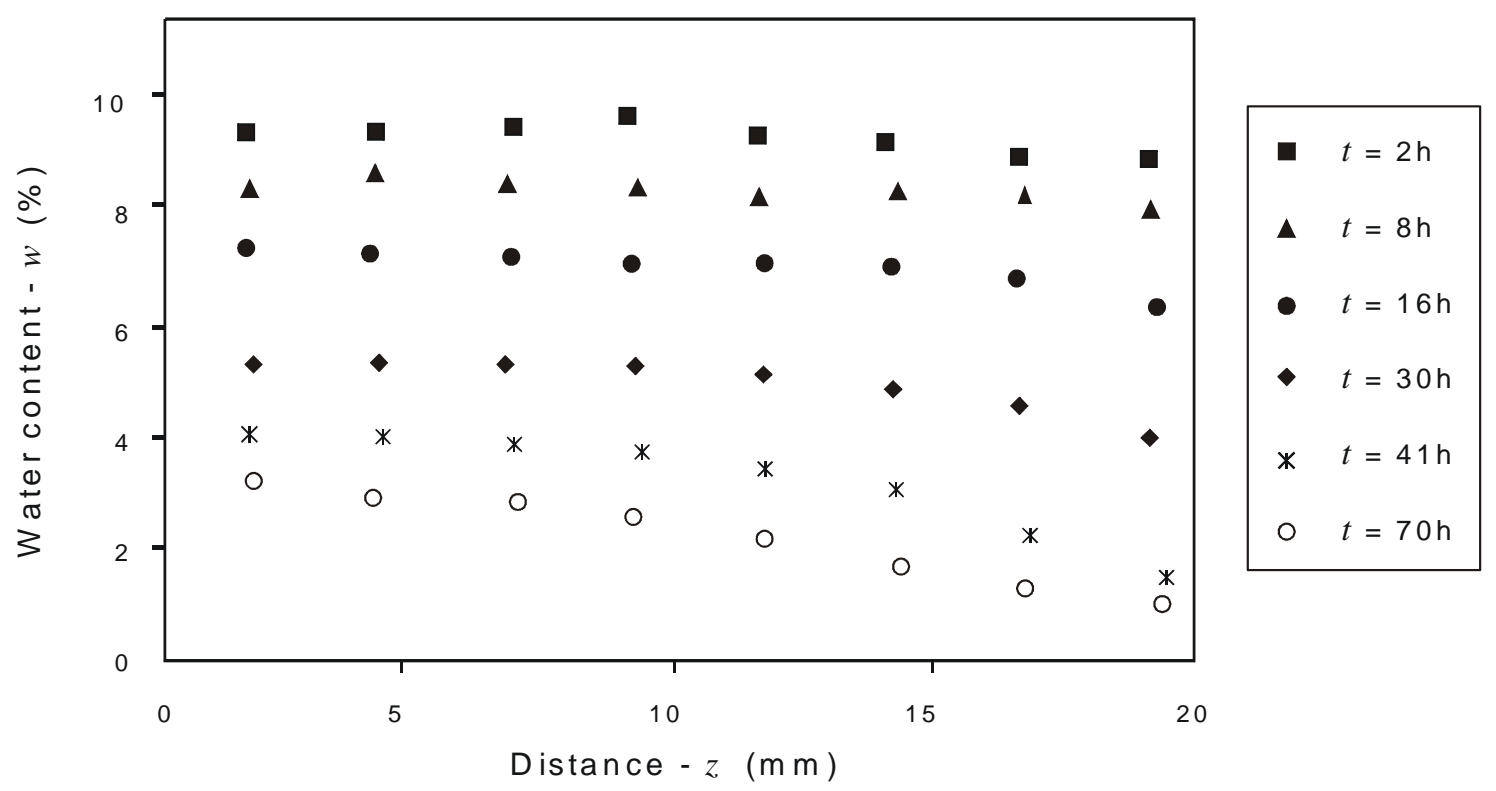

Figure 4: First preliminary experiment: Evolution of the soil sample water content profiles in the drying atmosphere $(R H=33 \%)$. 


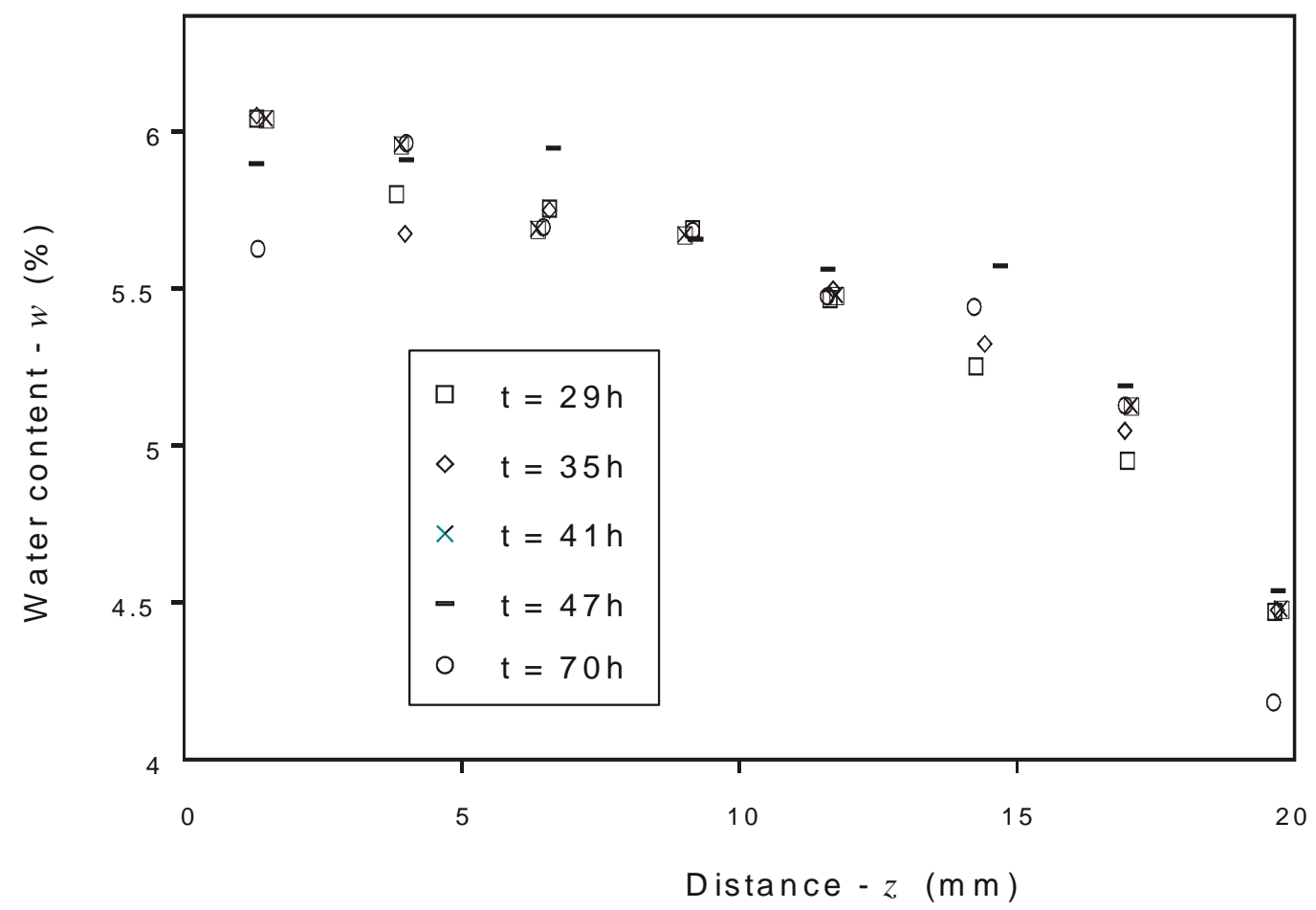

Figure 5: First preliminary experiment: Evolution of the soil sample water content profiles in the saturated atmosphere $(R H=100 \%)$. 


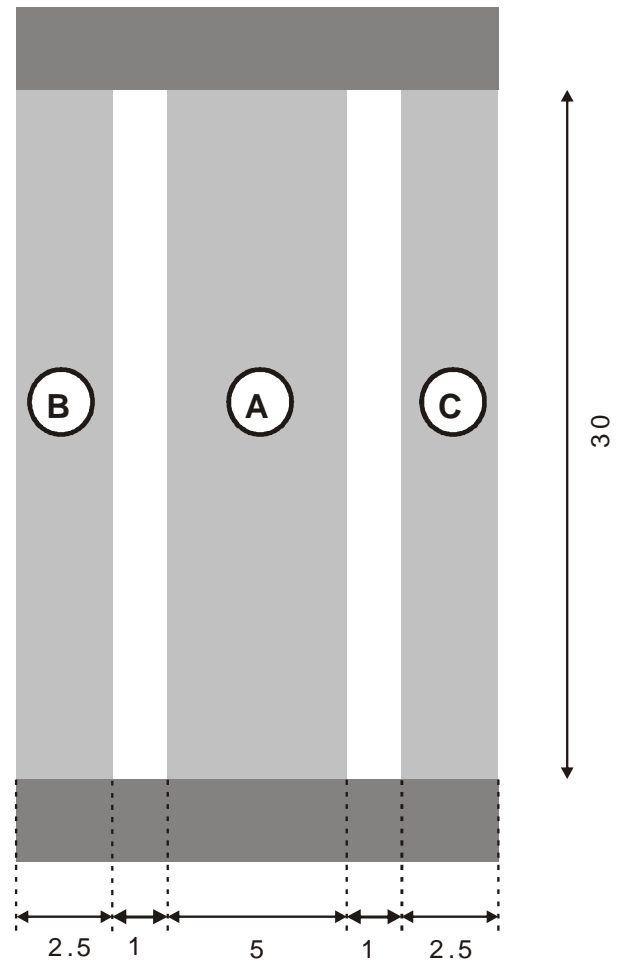

Figure 6: Second preliminary experiment: Schematic view of the experimental device (dimensions are given in $\mathrm{cm}$ ). 


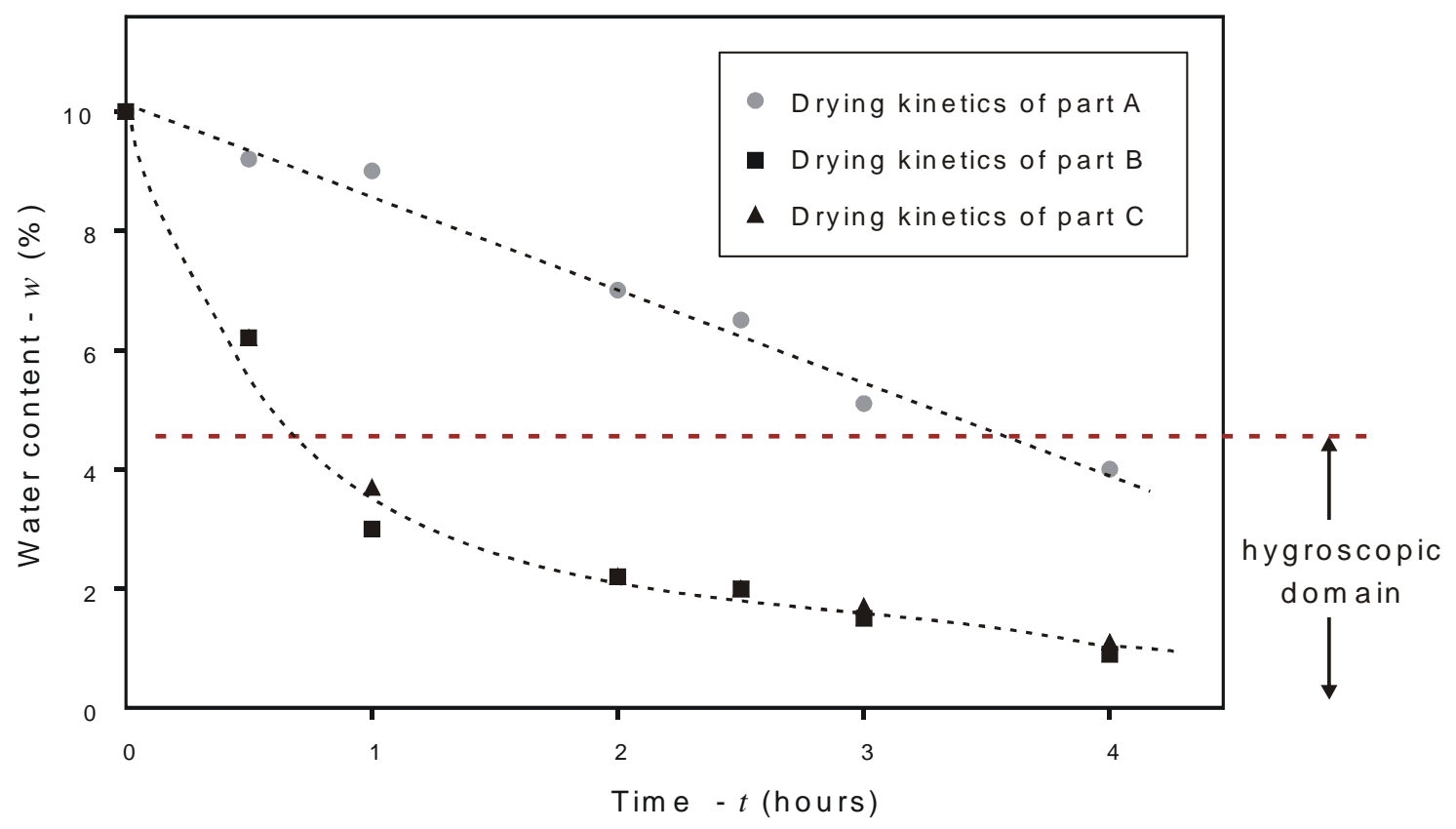

Figure 7: Second preliminary experiment: Drying kinetics of the three parts A, B and C. 


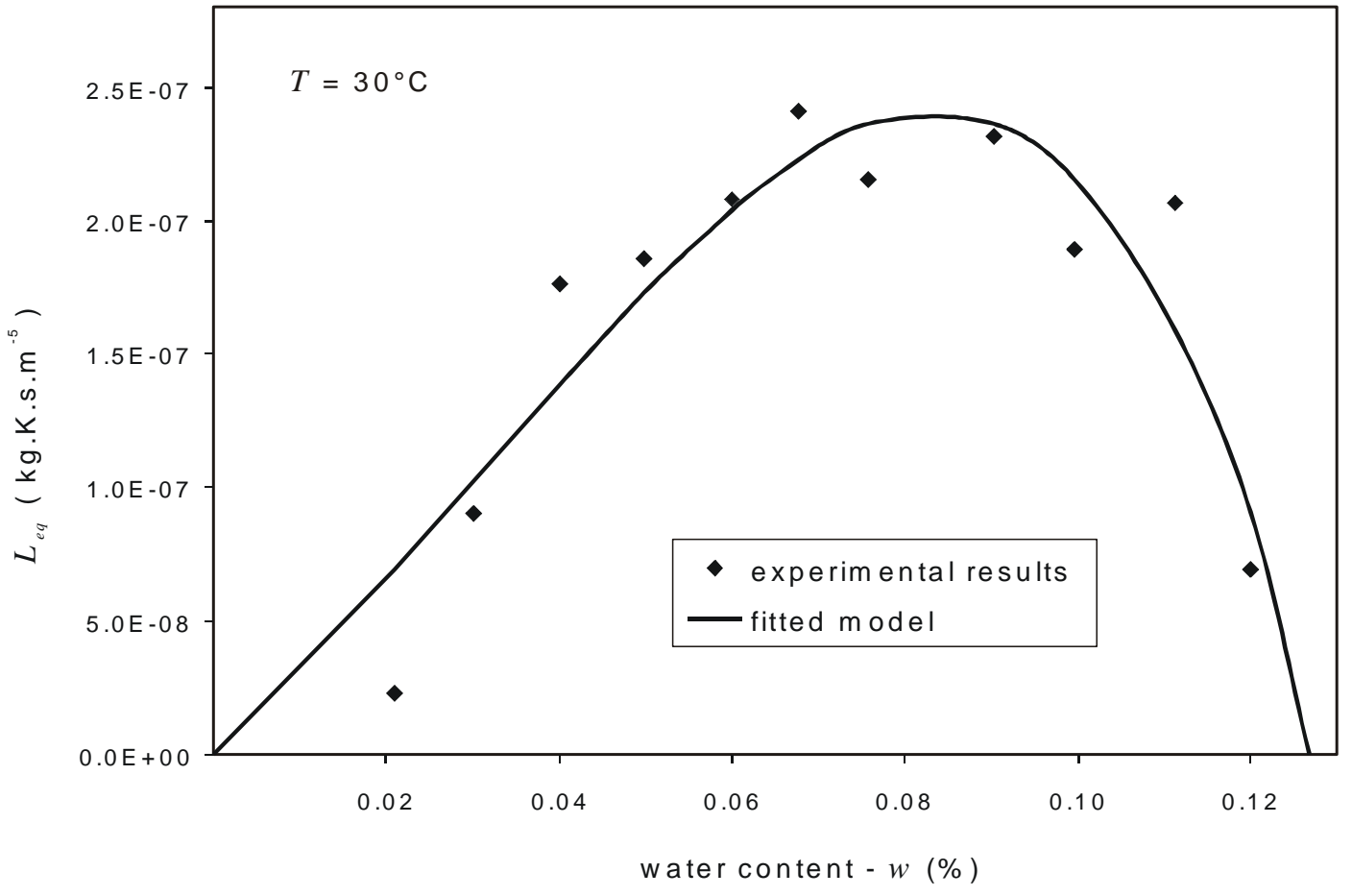

Figure 8: Phase change model (Eqs. 3-5): Variation of the phenomenological coefficient close to equilibrium $L_{e q}$ as a function of the water content $w$ at $T=30^{\circ} \mathrm{C}$. 


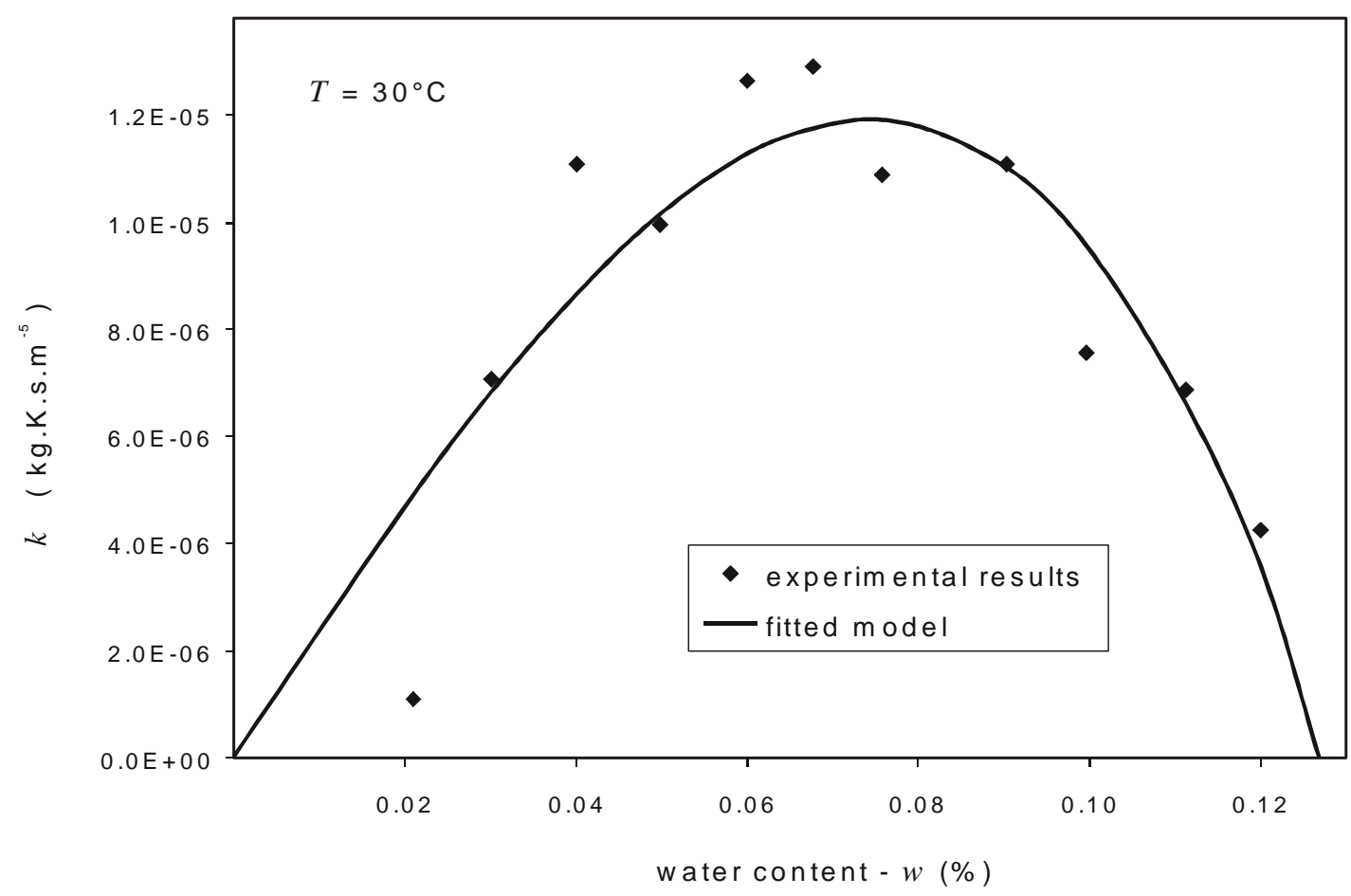

Figure 9: Phase change model (Eqs. 3-5): Variation of the parameter $k$ as a function of the water content $w$ at $T=30^{\circ} \mathrm{C}$. 


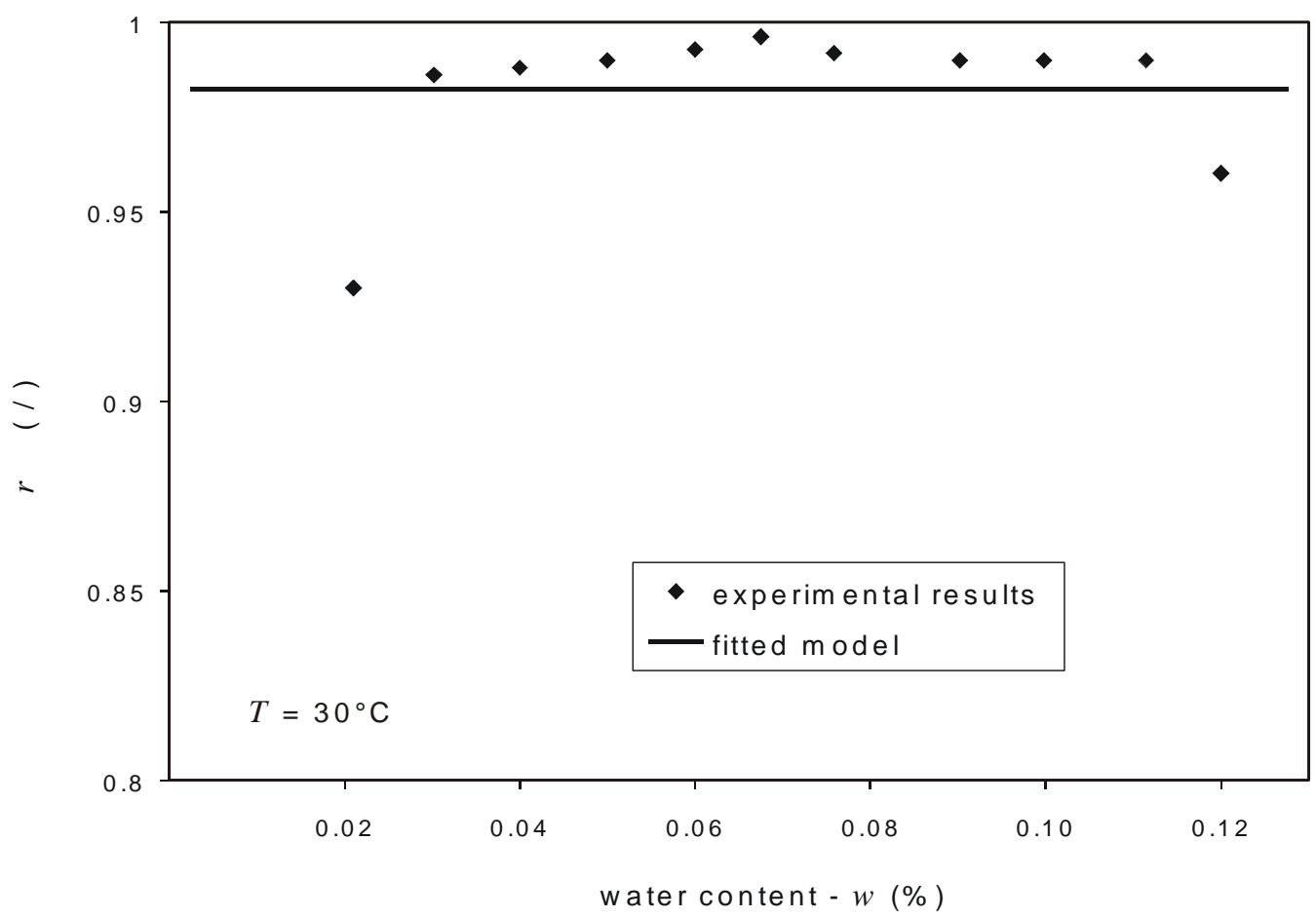

Figure 10: Phase change model (Eqs. 3-5): Variation of the transition criterion $r$ as a function of the water content $w$ at $T=30^{\circ} \mathrm{C}$. 


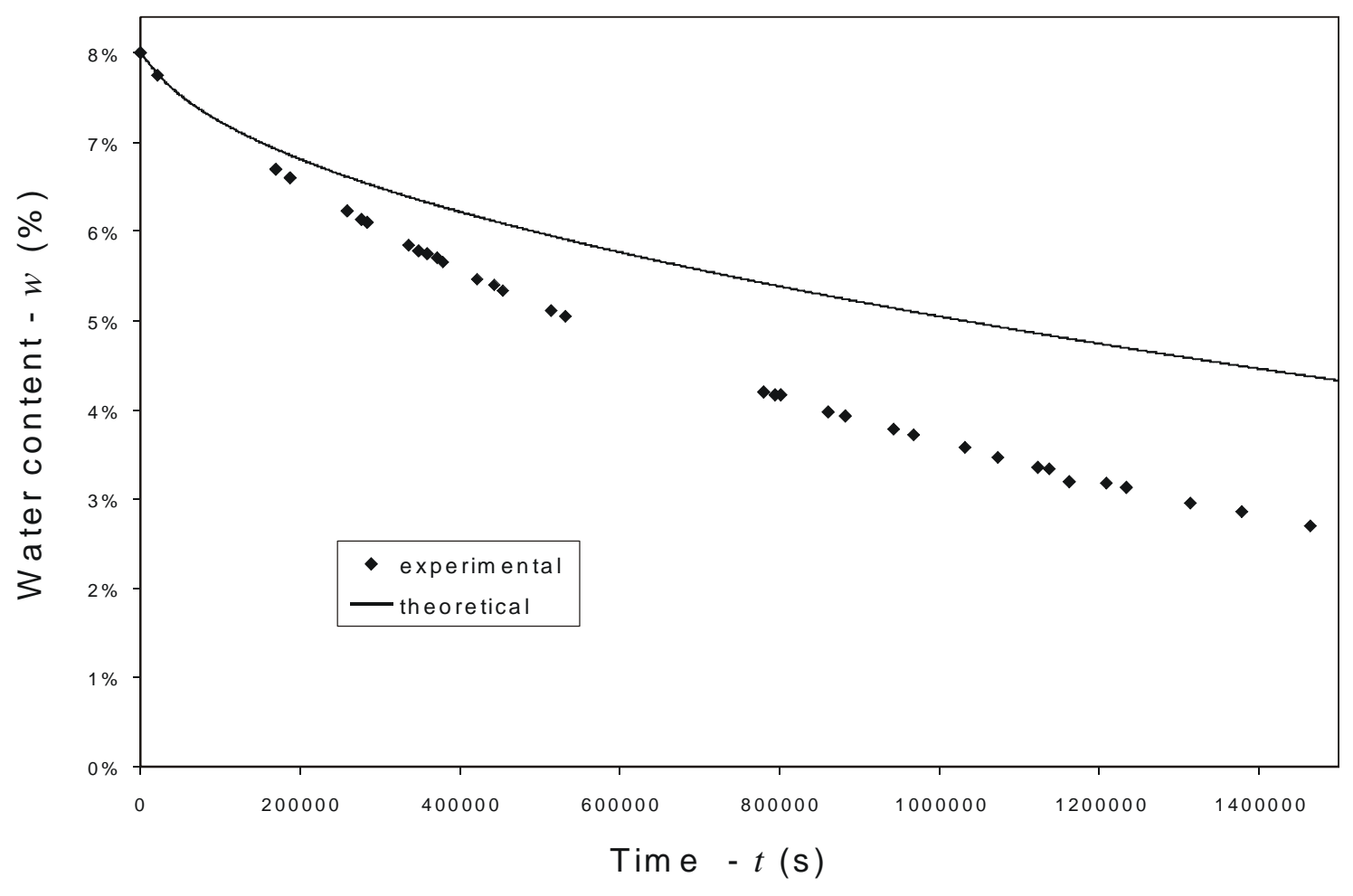

Figure 11: Comparison of experimental kinetics and theoretical prediction for a $10 \mathrm{~cm}$ soil column in drying conditions $(R H=30 \%)$. 